\title{
ACCOMPLISHING REUSE WITH A SIMULATION CONCEPTUAL MODEL
}

\author{
Osman Balci \\ James D. Arthur \\ Department of Computer Science \\ Virginia Tech \\ Blacksburg, VA 24061, USA
}

\author{
Richard E. Nance \\ Orca Computer, Inc. \\ 1800 Kraft Drive, Suite 111 \\ Blacksburg, VA 24060, USA
}

\begin{abstract}
Reuse has been very difficult or in some cases impossible in the Modeling and Simulation (M\&S) discipline. This paper focuses on how reuse can be accomplished by using a conceptual model $(\mathrm{CM})$ in a community of interest (COI). We address the issue of reuse in a multifaceted manner covering many areas (types) of $M \& S$ such as discrete, continuous, Monte Carlo, system dynamics, gamingbased, and agent-based. M\&S is commonly employed and reuse is critically needed by many COIs such as air traffic control, automobile manufacturing, ballistic missile defense, business process reengineering, emergency response management, military training, network-centric operations and warfare, supply chain management, telecommunications, and transportation. We present how a CM developed for a COI can assist in reuse for the design of any type of large-scale complex M\&S application in that COI. A CM becomes an asset for a COI and offers significant economic benefits through its effective reuse.
\end{abstract}

\section{INTRODUCTION}

A conceptual model $(\mathrm{CM})$ has been recognized as an integral part of Modeling and Simulation (M\&S) application development as the complexity and size of M\&S applications have continued to increase with the availability of more powerful computer systems (Arthur and Nance 2007; Balci and Ormsby 2007; Robinson et al. 2006; Robinson 2004, 2006, 2007).

Balci and Ormsby (2007) propose to use a CM to enable reuse at the highest level. The objective of this paper is to extend the Balci-Ormsby approach, noting the potential benefits and effectiveness of $\mathrm{CM}$ reuse in an M\&S application to a large, complex problem domain. The focus on reuse of a $\mathrm{CM}$ in the design of any type of largescale, complex M\&S application is driven by the recognized potential benefits: reduced development cost and time, effective use of subject matter expertise, increased quality (through the reuse of certified artifacts), and reduced risk. Reuse promotes the "do not reinvent the wheel" principle that seeks economies for sponsors or taxpayers.

Traditionally, reuse-based M\&S application development has been accomplished at the following levels:

- M\&S Programming-level Reuse

- M\&S Programming Frameworks-level Reuse

- M\&S Design-level Reuse

- M\&S Component-level Reuse

- M\&S Commercial-Off-The-Shelf (COTS) and Government-Off-The-Shelf (GOTS) Productslevel Reuse

- M\&S Application-level Reuse

- Network-Centric M\&S Application-level Reuse

Section 2 explains each of the levels, elaborating the difficulties encountered. Section 3 describes the role of a $\mathrm{CM}$ in reuse. Reuse-based M\&S application design using a $\mathrm{CM}$ is presented in Section 4. Section 5 provides conclusions.

\section{LEVELS OF REUSE IN M\&S APPLICATION DEVELOPMENT}

At the programming level, classes (under the objectoriented paradigm) and subroutines/functions (under the procedural paradigm) are extracted from a library using an Integrated Development Environment (IDE) such as Microsoft Visual Studio. However, reuse at this level is extremely difficult due to the many options in programming languages (e.g., C, Fortran, C++, Java), differences in operating systems (e.g., Unix, Windows), and variations among hardware platforms (e.g., Intel, Sparc) supporting language translators. An artifact programmed in Fortran under the Solaris Unix operating system on a Sun Sparc workstation cannot be easily reused in an M\&S application being developed in $\mathrm{C}++$ under the Windows XP Professional operating system on an Intel-based workstation. 
M\&S programming frameworks are categorized under the Object-Oriented Paradigm (OOP) and Procedural Paradigm (PP). Balci (1988) describes four conceptual frameworks under PP for simulation programming in a highlevel programming language: event scheduling (ES), activity scanning (AS), three-phase approach (TPA), and process interaction (PI). A simulation programmer is guided under one of these frameworks by reusing the concepts underlying that conceptual framework. However, an artifact programmed under one framework cannot be easily reused under another.

Reuse at the design level is feasible if the same design paradigm is employed for both the M\&S application development and the reusable work products. The reuse is also affected by the design patterns employed. For example, an M\&S application being designed under the objectoriented design (OOD) approach can reuse work products created under the OOP. Unified Modeling Language (UML) diagrams are provided as an international standard to describe an OOD. UML diagrams assist an M\&S designer in understanding and reusing an existing OOD.

However, reuse at the design level is also extremely difficult since it requires the reuse of the same design paradigm. Many types of M\&S applications exist such as discrete, continuous, Monte Carlo, system dynamics, agentbased, and gaming-based. Simulation model representation is completely different for each of the above types of M\&S applications. For example, a continuous simulation model consists of differential equations, and the OOP is not applicable for reuse. Monte Carlo simulation is based on statistical random sampling. A system dynamics simulation model represents cause-and-effect relationships in terms of causal-loop diagrams, flow diagrams with levels and rates, and equations. An agent-based simulation model represents agents and their interactions. An agent is "intelligent", adaptive, autonomous, goal/self-directed, has the ability to learn, and can change its behaviors based on experience. Different types of simulation models are designed under different paradigms, and one paradigm cannot be easily accommodated within another. Yilmaz and Ören (2004) present a CM for reusable simulations under the conceptual framework of a model-simulator-experimental frame.

M\&S component level reuse is intended to enable the assembly (composition) of a simulation model by way of employing already developed model components in a similar fashion as an automobile is assembled from previously produced parts. A component may correspond to a submodel or a model module. Reuse at this higher level of granularity reduces development time and cost over that of reuse at the class or function level. However, this approach to reuse is also extremely difficult since each reusable component can be implemented in a different programming language intended to run under a particular operating system on a specific hardware platform.
M\&S COTS (e.g., Arena, AutoMod, and OpNet) and GOTS products enable reuse of components within their IDEs. Such an IDE provides a library of reusable model components. A user can click, drag, and drop an already developed component from the library and reuse it in building a simulation model. However, such reuse is specific only to that particular COTS or GOTS IDE, and portability to another IDE would become a user responsibility.

Reuse at the application level is feasible if the Intended Uses (objectives) of the reusable M\&S application match the Intended Uses of the M\&S application under development. For example, the Department of Defense (DoD) provides a Modeling and Simulation Resource Repository (MSRR) (DoD 2008) containing previously developed M\&S applications. Some of these applications are independently certified for a set of Intended Uses. Some are not well documented and come in binary executable form only. Even if the source code is provided, understanding the code sufficiently to modify the represented complex behavior is extremely challenging. Reusability of earlier developed M\&S applications is dependent on the runtime environment compatibility and the match between Intended Uses.

A network-centric M\&S application describes the case in which the M\&S components interoperate with each other over a network (e.g., Internet, virtual private network, wireless network). Such an application consists of a federation of simulation models or model components interconnected over a network typically for the purpose of training geographically dispersed persons or groups.

High Level Architecture (HLA) is a DoD, IEEE, and NATO standard for developing a network-centric M\&S application by way of reuse of simulation models distributed over a network (IEEE 2000). HLA enables the interoperability of a federation of simulation models running on different nodes of a network. If a simulation model is built in compliance with the HLA standard, then that model can be reused by other models interconnected through the HLA protocol over a network.

Service Oriented Architecture (SOA) is yet another architecture based on the industry standard web services and eXtensible Markup Language (XML). SOA can be employed for developing a network-centric M\&S application by way of reuse of simulation models, submodels, components, and services over a network. For example, Sabah and Balci (2005) provide a web service for random variate generation (RVG) from 27 probability distributions with general statistics, scatter plot, and histogram of the requested random variates. The RVG web service can be called from any $M \& S$ application that runs on a server computer over a network using XML as the vehicle for interoperability. Reuse and interoperability are fully achieved regardless of the programming language, operating system, or hardware platform. However, this type of 
reuse is possible only for network-centric or web-based M\&S application development.

\section{THE ROLE OF A CM IN REUSE}

A simulation Conceptual Model is a repository of highlevel conceptual constructs and knowledge specified in a variety of communicative forms (e.g., animation, audio, chart, diagram, drawing, equation, graph, image, text, and video) intended to assist in the design of any type of largescale complex M\&S application. The targeted "re-users" can include $M \& S$ application designers, $M \& S$ project managers, and $M \& S$ analysts.

Many different types of M\&S applications exist including discrete, continuous, Monte Carlo, system dynamics, gaming-based, agent-based, artificial intelligencebased, and virtual reality-based. Different types of $M \& S$ applications are commonly employed in a Community of Interest (COI) such as air traffic control, automobile manufacturing, ballistic missile defense, business process reengineering, emergency response management, military training, network-centric operations and warfare, supply chain management, telecommunications, and transportation. Reuse is critically needed to facilitate the design of any type of large-scale complex M\&S application in a particular COI.

We propose the development of a CM by following the life cycle presented by Balci and Ormsby (2007) to enable reuse independently of the M\&S application type, M\&S application design strategy, and M\&S application implementation (programming) platform. The proposed CM should be built as a repository containing high-level conceptual constructs and knowledge for a particular COI (problem domain) at the highest level of abstraction as depicted in Figure 1.

We believe that the highest degree of applicability of reuse can be achieved by providing COI (problem domain)-specific conceptual constructs and knowledge at the highest level of abstraction in a CM. The conceptual constructs and knowledge provided in a CM can be reused by the designer of any type of M\&S application in the COI. Such a CM provides many roles and benefits including (Balci and Ormsby 2007):

1. assist in designing not just one $M \& S$ application but many in a particular COI (problem domain),

2. assist in designing any type of $M \& S$ application,

3. enable reuse at the highest degree of applicability,

4. enable effective communication among the people involved in a large-scale M\&S project such as stakeholders, potential users, managers, analysts, and $\mathrm{M} \& \mathrm{~S}$ developers,

5. assist in overcoming the complexity of designing large-scale complex M\&S applications in a COI,
6. provide a multimedia knowledge base covering the areas of expertise needed for designing largescale complex M\&S applications in a COI,

7. help a subject matter expert (SME) involved in an M\&S project to understand another SME's work,

8. facilitate the collaboration among the SMEs for designing a large-scale complex M\&S application in a COI,

9. assist in verification, validation, and certification (VV\&C) of the M\&S application,

10. support effective and efficient VV\&C of the M\&S application,

11. assist in the specifications of test designs, test cases, and test procedures for the M\&S application,

12. guide the managers, analysts, and developers in M\&S application design in a COI,

13. assist in proper formulation of $M \& S$ application Intended Uses (objectives),

14. assist in the generation of new M\&S requirements, and

15. provide significant economic benefits through reuse.

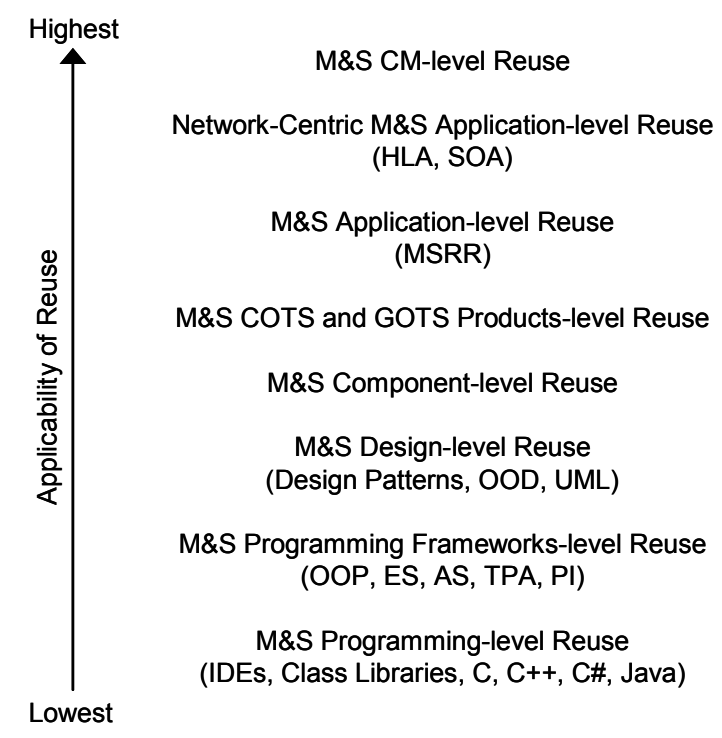

Figure 1: Reuse levels versus applicability

\section{REUSE-BASED M\&S APPLICATION DESIGN USING A CM}

Following the life cycle presented by Balci and Ormsby (2007), a CM can be developed to provide the roles and benefits stated above. These benefits are realized within a particular COI. To illustrate the envisioned approach to $\mathrm{CM}$ reuse that can perform the roles and achieve the benefits cited above, we use a suitably complex example: the COI called Emergency Response Management (ERM). 
Disasters are typically categorized as man-made and natural. Man-made disasters include biological, critical infrastructure (e.g., bridge, building, power plant, depot) destruction, chemical, fires, and nuclear disasters, created accidentally or intentionally (terrorist activities). Natural disasters include earthquakes, epidemics, floods, hurricanes, tornadoes, and tsunamis.

Based on the U.S. National Response Plan (HLS 2004), states and cities in the U.S. are expected to have an ERM plan. Under a given ERM plan, first responders, decision makers, authorities involved, and citizens are expected to be trained.

M\&S is the only effective approach for assessing the operational effectiveness of a given ERM plan as well as for training. Anyone can develop a plan; but, the challenge is to make sure that the plan is operationally effective in responding to an incident. Live exercises, e.g., (VERTEX 2007), are performed to try out a given plan, but such exercises are limited in scope and do not provide the technical assurance required in assessing the operational effectiveness of a plan and performing training under all possible scenarios. Therefore, $\mathrm{M} \& \mathrm{~S}$ is most commonly used in the ERM COI for plan assessment and training.

For example, U.S. Joint Forces Command (USJFCOM 2008) leads the Noble Resolve experimentation campaign plan to enhance homeland defense and improve military support to civil authorities in advance of and following natural and man-made disasters. The Noble Resolve is mostly an M\&S-based experimentation.

To achieve the maximum potential for reuse, the CM should promote reapplication of ERM conceptual constructs and knowledge in the design of any type of M\&S application for two purposes: (a) operational effectiveness assessment of the ERM plan, and (b) simulation-based training of the people based on the ERM plan. Since reuse potential extends to hundreds of cities and states, there exist hundreds of ERM plans, requiring the development of hundreds of M\&S applications for the above purposes. If a $\mathrm{CM}$ were made available for this COI, designers of hundreds of M\&S applications would realize the benefits stated in Section 3 and "re-inventing the wheel again and again" would be prevented resulting in significant economic gains.

COI problem domain SMEs should be employed in specifying the content of the CM as depicted in Figure 2. Problem domain-specific authoritative expertise should be used in specifying the CM's conceptual constructs and knowledge. For example, in the ERM COI, SMEs can specify:

1. how best to equip a Hazardous Materials Response Team depending on the type of incident,

2. most effective strategies for evacuating an area in response to a particular disaster,

3. most effective strategies for search and rescue operations in a particular incident,
4. existing and emerging technologies (e.g., sensors, surveillance cameras, unmanned vehicles, wireless devices) that can be employed for responding to a particular emergency incident,

5. most up-to-date knowledge about possible manmade disasters such as chemical or biological,

6. most effective responses to a particular disaster based on past experience,

7. best practices for accomplishing interoperability among the emergency response authorities who have jurisdiction over different geographical areas,

8. how to quickly establish an Internet Protocol (IP)based wireless communication among the first responders, decision makers, and citizens, when the existing communication systems go down as a result of a disaster,

9. case studies on past disasters documenting lessons learned, and

10. concepts of emergency response operations.

The conceptual constructs and knowledge specified by SMEs in the CM can be reused by M\&S application designers in the design of many M\&S applications of many types as depicted in Figure 2.

We propose that the lead organization in the COI sponsor the development and maintenance of the CM and make it available for use by all M\&S application developers in that COI. Example lead organizations include Federal Emergency Management Agency (FEMA) in the ERM COI, Missile Defense Agency (MDA) in the ballistic missile defense COI, National Institute of Standards and Technology (NIST) in the manufacturing systems COI, U.S. Department of Transportation in the transportation COI, Federal Aviation Administration (FAA) in the air traffic control COI, and U.S. Department of Defense in the simulation-based training COI.

The CM should be a live resource continuously updated to reflect the most up-to-date authoritative expertise and should be provided over the Internet for easy access by geographically dispersed M\&S application designers in the COI for which the CM serves. The CM should be developed as a multimedia repository to enable effective reuse of its content.

Another option is to provide the $\mathrm{CM}$ as a wiki. A wiki is a collaborative website designed to enable anyone who has access rights to contribute or modify the website's content. A wiki type of CM would open up the CM content for contribution or modification by all those people who consider themselves to be SMEs in that COI. Although the acquisition of knowledge is greatly facilitated with a wiki, the quality control becomes a serious challenge. If the lead organization/sponsor of the CM controls who can contribute or modify the CM content and establishes some kind of 


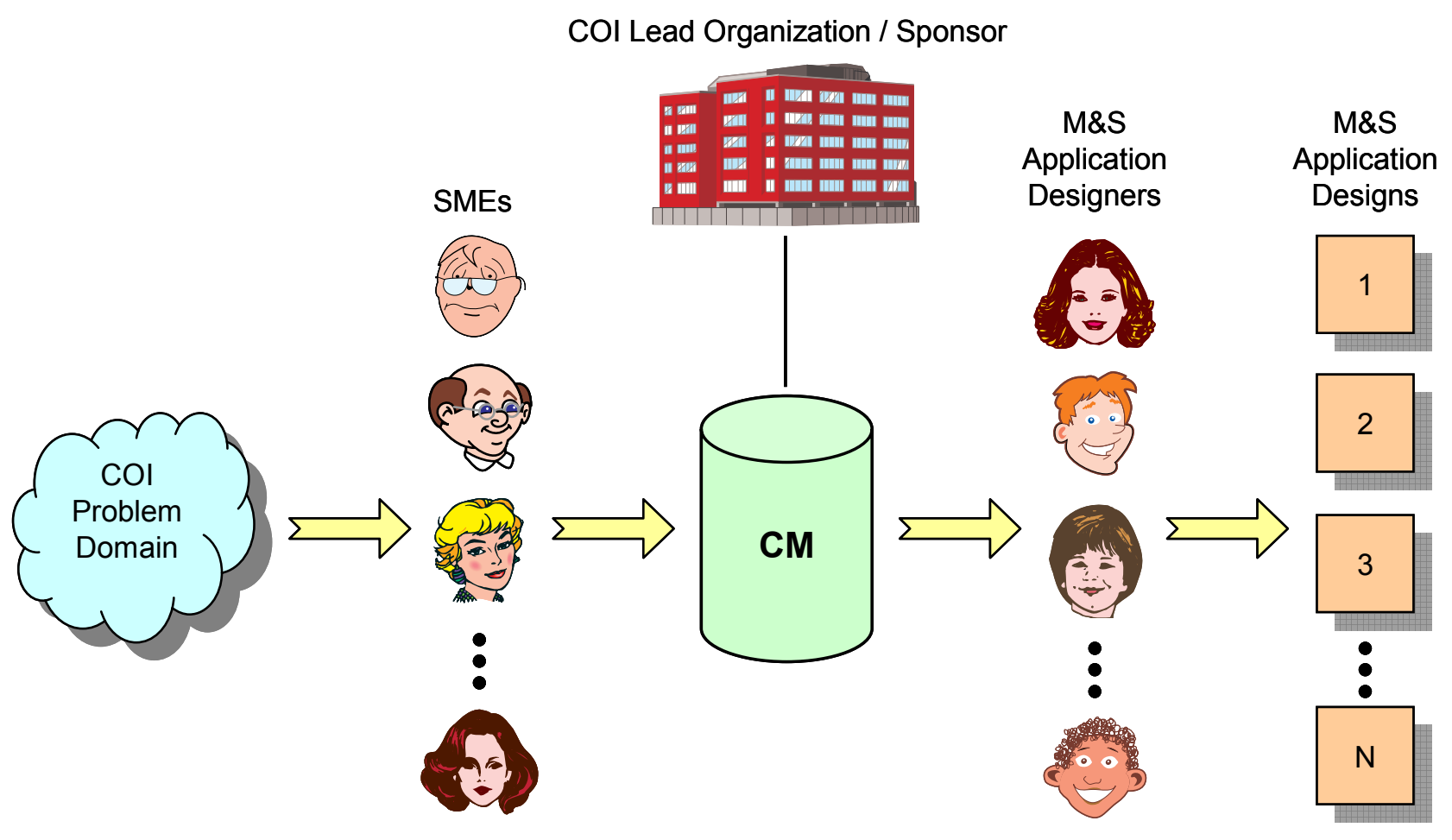

Figure 2: Creation and use of a $\mathrm{CM}$ in a COI

peer review, then a wiki type of CM might become feasible.

\section{CONCLUSIONS}

The stated objective of this paper is to extend the BalciOrmsby approach (Balci and Ormsby 2007) to CM reuse, noting the potential benefits and increased effectiveness derived in large-scale, complex M\&S applications. This objective is realized in specific terms through:

- advancement of the concept of CM reuse in largescale, complex M\&S applications within a COI, following the Balci-Ormsby approach,

- identification of the difficulties inherent in reuse within M\&S applications, considering the multiplicity in types and the levels of modeling granularity,

- explanation of the significant challenges of reuse at the differing levels of model abstraction, and

- description of an application of the Balci-Ormsby approach to effect CM reuse in ERM.

A lead organization is strongly recommended to fund the development and continuous update of a $\mathrm{CM}$ within its
COI for reuse by M\&S application designers in that COI. Return on investment shall be realized by preventing "reinventing the wheel again and again." Economic benefits shall be gained by all COI companies and organizations in developing complex M\&S applications. A CM should be a "live" repository continuously updated to reflect up-to-date knowledge. CM content quality should be assured and the wiki approach with no peer review should be avoided. CM content should be generically applicable for any type of M\&S application under any design strategy to assure its wider reuse. The content should be useful not only for M\&S application designers, but also for managers, analysts, and stakeholders.

\section{REFERENCES}

Arthur, J. D., and R. E. Nance. 2007. Investigating the use of software requirements engineering techniques in simulation modeling. Journal of Simulation 1: 3 (Aug.) 159-174.

Balci, O. 1988. The implementation of four conceptual frameworks for simulation modeling in high-level languages. In Proceeding of the 1988 Winter Simulation Conference, ed. M. A. Abrams, P. L. Haigh, and J. C. Comfort, 287-295. Piscataway, New Jersey: Institute of Electrical and Electronics Engineers, Inc. 
Balci, O., and W. F. Ormsby. 2007. Conceptual modeling for designing large-scale simulations. Journal of Simulation 1: 3 (Aug.) 175-186.

DoD. 2008. Modeling and Simulation Resource Repository. Department of Defense, http://msrr.dmso.mil/

HLS. 2004. National Response Plan. U.S. Department of Homeland Security, Washington, D.C., Dec., http://www.scd.state.hi.us/docume nts/nrp.pdf

IEEE. 2000. IEEE Standard for Modeling and Simulation (M\&S) High Level Architecture (HLA) - Framework and Rules. IEEE Standard No. 1516-2000. Piscataway, New Jersey: Institute of Electrical and Electronics Engineers, Inc.

Robinson, S. 2004. Simulation: The Practice of Model Development and Use. West Sussex, England: John Wiley \& Sons Ltd.

Robinson, S. 2006. Issues in conceptual modelling for simulation: setting a research agenda. In Proceedings of the 2006 Operational Research Society Simulation Workshop, eds. S. Robinson, S. Taylor, S. Brailsford, and J. Garnett. 165-174. Birmingham, England: Operational Research Society.

Robinson, S. 2007. The future's bright the future's...Conceptual modelling for simulation! Journal of Simulation 1: 3 (Aug.) 149-152.

Robinson, S., S. Taylor, S. Brailsford, and J. Garnett, eds. 2006. Proceedings of the 2006 Operational Research Society Simulation Workshop (Lemington Spa, UK, March 28-29). Birmingham, England: Operational Research Society. http://arthur.cs.vt.edu/conceptua l-modelling/

Sabah, M., and O. Balci. 2005. Web-based random variate generation for stochastic simulations. International Journal of Simulation and Process Modelling 1: 1-2, $16-25$.

USJFCOM. 2008. The Noble Resolve Campaign. U.S. Joint Forces Command (USJFCOM), Norfolk, VA. http://www.jfcom.mil/about/experi ments/nobleresolve.html

VERTEX. 2007. Virginia Emergency Response Team Exercise (VERTEX) 2007 Functional Exercise. Commonwealth of Virginia, Richmond, VA.

Yilmaz, L., and T. I. Ören. 2004. A conceptual model for reusable simulations within a model-simulator-context framework. In Proceedings of the Conference on Conceptual Modelling and Simulation (Part of the I3M International Mediterranean Modelling Multiconference, Genoa, Italy, Oct. 28-31). 235-241.

\section{AUTHOR BIOGRAPHIES}

OSMAN BALCI is a Professor of Computer Science at Virginia Polytechnic Institute and State University (Virginia Tech). He received B.S. and M.S. degrees from Boğaziçi University (Istanbul) in 1975 and 1977, and M.S. and Ph.D. degrees from Syracuse University (New York) in 1978 and 1981. Dr. Balci served as the Editor-in-Chief of two international journals: Annals of Software Engineering, 1993-2002 and World Wide Web, 1996-2000. He currently serves as ACM SIGSIM Chair; Area Editor of $A C M$ Transactions on Modeling and Computer Simulation, and Modeling and Simulation (M\&S) Category Editor of $A C M$ Computing Reviews. He also serves on the Editorial Boards of Journal of Simulation and International Journal of Simulation and Process Modeling. He served as an elected Director at Large of the Society for M\&S International for two terms, 2002-2006. Most of Dr. Balci's work has been funded by the U.S. Navy since 1983. From 1998 to 2004, he provided technical services for the U.S. Missile Defense Agency programs in the areas of complex M\&S acceptability assessment and complex system independent verification and validation (IV\&V). His current areas of expertise center on network-centric software engineering; networkcentric system architecting; M\&S; software IV\&V; V\&V, testing, certification, and quality assessment of models and simulations. His e-mail and web addresses are balcievt.edu and http://manta.cs.vt.edu/balci.

JAMES D. ARTHUR is an Associate Professor of Computer Science at Virginia Tech. He received B.S and M.A. degrees in Mathematics from the University of North Carolina at Greensboro in 1972 and 1973, M.S. degree in Computer Science from the University of Houston in 1979, and M.S. and Ph.D. degrees in Computer Science from Purdue University in 1981 and 1983. His research interests include Software Engineering (Requirements Engineering, Methods and Methodologies supporting Software Quality Assessment, and IV\&V Processes), Parallel Computation, and User Support Environments. Dr. Arthur is the author of over 75 papers on software engineering, requirements engineering, software quality assessment, IV\&V, and user/machine interaction. He has served as: a participating member of IEEE Working Group on Reference Models for V\&V Methods; an advisor to the U.S. Navy Commonality Working Group; Chair of Education Panel for National Software Council Workshop; Guest Editor for Annals of Software Engineering special volume on Process and Product Quality Measurement; and Principal Investigator or Investigator on 19 externally funded research projects totaling in excess of $\$ 3.5$ million. His e-mail and web addresses are arthurevt.edu and http://people.cs.vt.edu/ arthur/. 
RICHARD E. NANCE is an Emeritus Professor of Computer Science at Virginia Tech and Chief Scientist, Orca Computer, Inc. He received B.S. and M.S. degrees from N.C. State University in 1962 and 1966, and the Ph.D. degree from Purdue University in 1968. He has served on the faculties of Southern Methodist University and Virginia Tech, where he was Department Head of Computer Science, 1973-1979. Dr. Nance held research appointments at the Naval Surface Weapons Center (1979-80) and at the Imperial College of Science and Technology (UK). Within ACM, he has chaired two special interest groups: Information Retrieval (SIGIR), 1970-71 and Simulation (SIGSIM), 1983-85. He is the author of over 150 papers on discrete event simulation, performance modeling and evaluation, computer networks, and software engineering. Dr. Nance has held several editorial positions and was the founding Editor-in-Chief of the ACM Transactions on Modeling and Computer Simulation, 1990-1995. He served as Program Chair for the 1990 Winter Simulation Conference. Dr. Nance has received several awards for his editorial and professional contributions, most recently the Lifetime Professional Achievement Award from the INFORMS Simulation Society. In 1996, he was named an ACM Fellow. In 2006 he was designated by the faculty as a Distinguished Alumnus of the Edward P. Fitts Department of Industrial \& Systems Engineering, North Carolina State University, He is a member of Sigma Xi, Alpha Pi Mu, Upsilon Pi Epsilon, ACM, and INFORMS. His e-mail address is nancedvt.edu. 